\title{
DE LA NÉCESSITÉ DE DÉSORDRE DANS LA DÉMOCRATIE
}

\author{
Author(s) / Auteur(s) : \\ Claude LAMBERT \\ Président de $S \& O A S B L$, Vice-Président de l'UES \\ Formateur G.I.R.O.S. (Systémique des organisations) \\ cepehello@gmail.com
}

\begin{abstract}
Résumé :
Dans cet article, je propose de m'appuyer sur une évaluation de dysfonctionnement démocratique partagée par de nombreux citoyens. Pour ce faire, je propose d'appliquer la conjecture de Heinz Von Foerster à la société contemporaine. La conjecture de von Foerster décrit le rapport de causalité circulaire entre une totalité (par exemple, une collectivité humaine) et ses éléments (les individus qui la composent). Elle établit que plus les relations inter-individuelles sont "rigides" plus le comportement de la totalité apparaîtra aux éléments individuels qui la composent comme doté d'une dynamique propre qui échappe à leur maîtrise alors qu'elle sera d'autant plus prédictible par un observateur extérieur.

C'est ainsi que je montrerai comment les relations entre les acteurs de nos sociétés sont dominées par le paradigme de l'échange marchand basé sur la quantification, l'équivalence et la liberté des acteurs. D'un point de vue éthique, on ne peut que se féliciter de la liberté ainsi permise. Au-delà du contenu de ce type de relation, je fais l'hypothèse qu'elles ont par nature un caractère rigide et prédictible dans la forme, rencontrant ainsi les conditions d'application de la conjecture de H. von Foerster. Ceci se manifeste par un sentiment régulièrement partagé que la totalité est guidée ou manipulée par un "pouvoir obscur" ou "main invisible". A la recherche de sens, cette perception alimente et justifie les discours populistes et les théories du complot. Cependant, on ne peut se satisfaire de cette explication en invitant les acteurs de l'intérieur à adopter le point de vue de l'observateur extérieur. Les deux objectivités - de l'intérieur et de l'extérieur - se valent selon le point de vue. Il est donc utile de rester au niveau du monde-vécu et d'explorer un angle de vue alternatif. C'est ainsi que l'on peut mettre en rapport la liberté individuelle permise par le marché vis-à-vis de la liberté d'engagement dans la vie sociale. Cette dernière est rencontrée au sein d'une communauté animée par le paradigme du don-contredon au sens de Marcel Mauss. Le paradigme du don se manifeste aujourd'hui dans les formes d'associations informelles : associations de voisins, réseaux d'échanges, mouvements de militance morale... La relation qui s'établi par le don a un caractère imprédictible et incertain qui tranche avec la "simplicité" et univocité de la relation marchande. Ce caractère incertain du don-contredon introduit dans la société de l'inattendu, ce qui d'un point de vue systémique est favorable à l'innovation et l'adaptation. On ne peut alors que s'inquiéter de la tendance actuelle à la marchandisation du service public, la professionnalisation du service aux personnes, à l'application des critères du marché au fonctionnement des associations du secteur non-marchand... Cette tendance représente une réduction de la complexité sociale favorable à la rigidification des relations. Il ne s'agit pas ici de faire le procès du paradigme de l'échange marchand car il assure l'indépendance et l'accès à l'étranger au réseau d'échange. Il s'agit de suggérer une multiplicité des typologies de relations. C'est ainsi que d'un point de vue systémique, on ne peut que se féliciter d'une certaine forme de désordre social. Le régime démocratique accueille par définition conflit, débat et diversité de comportements.
\end{abstract}

Keywords / Mots-clés :

Liberté, désordre, adaptation, institution, association

\section{Systémique et systèmes humains}

Il peut être utile de signaler ou rappeler que la cybernétique et la systémique des années 1950 ont donné l'illusion de la possibilité de la commande et du contrôle. Initialement appliquées aux systèmes "mécaniques", la tentation d'extrapolation aux systèmes vivants et humains à très rapidement montré ses limites. Ces premières théories ne sont plus considérées aujourd'hui que contextuellement adaptées à des systèmes fermés ou mécaniques et sur une temporalité courte. D'un point de vue systémique, il 
est donc improbable de proposer un regard sur la démocratie qui serait orienté par le mythe d'un pouvoir centralisé. Des tentatives de gouvernement cybernétique ont montré leur limite (voir le projet chilien Cybersin ${ }^{1}$ ). Largement répondue, la théorie systémique et cybernétique doivent d'abord être comprise comme une grammaire commune à plusieurs disciplines scientifiques. Elles tendent à éclairer des phénomènes et des situations grâce à des principes communs partagés entre disciplines. Aujourd'hui les principes majeurs qui animent la théorie systémique sont ceux de l'auto-organisation et de l'autopoïèse (selon Varela), de la bifurcation et de l'émergence voire de l'incertitude, de l'incomplétude, ... . Dans ses derniers développements, la commande et le contrôle font place à des actions non-invasives et non-directives dès qu'il s'agit de systèmes complexes tel que les systèmes humains et sociaux selon les travaux du Prof. Gianfranco Minati².

Enfin, il est intéressant de préciser un aspect de l'application de la systémique dans le domaine du vivant et du social pour lequel la diversité des comportements au sein d'un système est un aspect essentiel. Cette diversité intervient dans la capacité d'adaptation de l'organisme ou de l'organisation immergé dans un contexte (voir : "la variété requise", selon R. Ashby). D’autre part, l'ouverture des frontières - ou membranes si l'on parle de cellule - est une condition du maintien d'un système dans le temps ; la fermeture des frontières entrainant le système à la disparition à moyen terme.

Auto-organisation, diversité, actions non-invasives sont autant de principes du vivant qui invite le systémicien à la prudence devant des approches centralisées et autoritaire lorsque l'on évoque la démocratie.

\section{Systémique dans le langage courant}

Dans le langage courant, le terme "système" et "systémique" sont régulièrement utilisés par la presse, les réseaux sociaux et par les représentants politiques. Pour le systémicien, il peut être irritant que cet usage soit réducteur voire péjoratif ou qu'il soit utilisé en vue de symboliser un dysfonctionnement social, économique ou politique. Parmi les usages courant, j'en relève deux : "crise systémique" et le "Système".

L'usage de l'expression "crise systémique" est facile à traduire en termes de principes systémiques. En effet, on peut traduire cela comme une combinaison de la circularité et de la (non-)régulation. On peut le comprendre par le fait qu'une impulsion sur un élément situé dans un ensemble d'éléments liés de manière circulaire se transmet de proche en proche dans la boucle rendant le fonctionnement hors de contrôle, à moins d'y ajouter une boucle de rétroaction négative.

Par contre, l'usage du terme "Système" peut s'interpréter pour celui qui l'utilise dans le sens d'un pouvoir caché qui renvoie à un sentiment d'impuissance de l'individu par rapport à une totalité. Dans ce sens, le terme sert comme la réification et l'identification d'un groupe responsable du dysfonctionnement social, économique ou politique. Si ce sentiment est partagé, il est légitime de ne pas le nier et de le respecter comme tel afin envisager les éventuelles réponses et implications du point de vue de la théorie des systèmes.

\section{Le "système" envisagé comme aliénation}

C'est ainsi que régulièrement nos démocraties souffrent d'un sentiment partagé par une part plus ou moins grande des citoyens qu'il existe au-delà du pouvoir démocratiquement élu, un ou plusieurs groupes plus ou moins secret. Ce ou ces groupes sont réellement aux commandes et manipule le fonctionnement afin de satisfaire ses intérêts au dépend de la population. Ce groupe peut être défini de manière diffuse sous des termes vagues dont celui de "système" ou identifié à une communauté d'intérêt ou d'obédience. Tour à tour, cela peut être les banques, un ou plusieurs lobbying, une

\footnotetext{
${ }^{1}$ https://fr.wikipedia.org/wiki/Projet_Cybersyn

${ }^{2}$ Minati, Gianfranco. Towards a Post-Bertalanffy Systemics. New York, NY: Springer Science+Business Media, 2015. P.211 $-217$
} 
communauté d'appartenance idéologique ou religieuse... C'est ainsi que ce sentiment donne lieu aux différentes théories dites du complot et sert d'argument à des mouvements populistes.

Je ne veux pas prétendre que le complot et le secret n'existe pas. Etant secret et inaccessible, je propose d'explorer une autre voie qui ouvre les possibilités de futur. En effet, si la manipulation était toujours vérifiée, elle ne donnerait aux citoyens que peu d'options si ce n'est la résignation, voire la violence visant à éliminer le ou les responsables.

\section{La systémique pour changer de point de vue}

Je propose un retournement du point de vue afin d'ouvrir de nouvelles perspectives tout en respectant sentiment partagé d'être guidé par un pouvoir obscur. C'est ainsi que je fais référence à Heinz Von Foerster et à la conjecture qui porte son nom. Elle s'énonce ainsi : "La conjecture de von Foerster décrit le rapport de causalité circulaire entre une totalité (par exemple, une collectivité humaine) et ses éléments (les individus qui la composent). Elle établit que plus les relations inter-individuelles sont "rigides" (plus l'influence sociale est forte) plus le comportement de la totalité apparaîtra aux éléments individuels qui la composent comme doté d'une dynamique propre qui échappe à leur maitrise alors qu'elle sera d'autant plus prédictible par un observateur extérieur.". ${ }^{3}$

Cette conjecture nous enseigne que dans une société, si les relations entre les acteurs ont un caractère mécanique ou trivial, alors les acteurs ont une perception du fonctionnement global qui s'autonomise comme mu par une "volonté" qui leur échappe. Il est alors légitime pour ces acteurs de vouloir attribuer ce fonctionnement qui leur échappe à un pouvoir extérieur qui se concrétise par la matérialisation d'un groupe quelconque. Il est naturel de donner du sens à ce sentiment et la démarche d'identifier le ou les coupables qui sont aux commandes est de bon sens. Cependant, si l'on suit la conjecture, le fonctionnement global n'est commandé par personne mais corrélé à la typologie de relation inter-individuelle, celle-ci s'inscrivant dans la relation circulaire qu'elle entretient avec la totalité.

Une analogie de ce phénomène peut être celui des essaims ou par exemple, le vol des étourneaux. Les travaux de Gianfranco Minati sur le concept de "Collective Beings"4 peut nous inspirer. L'hypothèse la plus courante et qui a fait l'objet de simulations est que les règles qui régissent les comportements des étourneaux sont simples et triviales en conformité avec l'exigence de la conjecture : une vitesse, une distance minimale et maximale par rapport à son voisin immédiat suffisent à expliquer le fonctionnement global de l'essaim.

A la différence des étourneaux, l'humain est un inventeur de sens et ne se contente pas de suivre le mouvement sans s'interroger sur la causalité des phénomènes qui l'entourent. A tout événement, il cherche à donner du sens : que ce soit par la recherche des causes, du coupable, par la recherche de l'utilité, .... Et si la logique et le raisonnement ne le permet pas il fera appel à la pensée magique. C'est ainsi que par respect pour ce besoin de donner du sens, il est utile de poursuivre l'exploration.

Mais alors quels seraient la nature des relations rigides ou triviales qui lient les acteurs au sein de la démocratie ? Cela est d'autant plus paradoxal de proposer que les relations "dominantes" entre les acteurs d'une démocratie puissent être rigides alors que la démocratie entend promouvoir la liberté individuelle. Le propos n'est pas ici de nier l'effectivité du respect de principe de la liberté

\footnotetext{
3 Andreewsky, Evelyne, and Robert Delorme, eds. Seconde Cybernétique et Complexité: Rencontres Avec Heinz von Foerster. Collection Ingénium. Paris: L'Harmattan, 2006. P.64

${ }^{4}$ Gianfranco Minati. Collective Beings. Contemporary Systems Thinking. Boston, MA: Springer US, 2006.
} 
individuelle mais de proposer de cadrer la conception de la liberté individuelle telle qu'elle est proposée au sein du paradigme dominant. En effet, la liberté individuelle est avant tout celle du marché économique selon ses principes fondateurs. Le postulat adopté par la majorité et qui n'est plus négocié que par peu d'acteurs est que les interactions entre les acteurs sont animées par la liberté des liens entre des acteurs agissant afin de satisfaire leurs intérêts. Cela s'exprime notamment par des expressions du type "win-win". Le gagnant-gagnant s'appuie sur des interactions entre acteurs qui vise à l'équivalence dans l'échange et à la comptabilisation de ce qui circule entre les acteurs. C'est ainsi que les acteurs, s'appuyant sur les postulats de l'échange marchand conservent leur liberté. En effet, une fois une transaction économique effectuée, il ne subsiste aucun lien entre les acteurs, ceci garantissant ainsi leur liberté. Animé par ce postulat, les liens entre acteurs sont non seulement distendus mais également prévisibles. C'est ainsi que je propose d'affirmer que la liberté définie selon le postulat du marché a pour conséquence la rigidification des relations entre les acteurs dans le sens de la prédictibilité et d'une rigidification croissante.

Si les relations entre les acteurs sont dominées par le paradigme de l'échange marchand, elles sont prévisibles. Si c'est le cas, cela corrobore l'application de la conjecture de Von Foester à une société démocratique qui est animé par la liberté entendue comme celle défendue par les postulats du marché.

Au point où nous en sommes, nous avons pris le point de vue de l'observateur extérieur tel que décrit par la conjecture. Nous pourrions donc nous arrêter ici et viser à diffuser ce message avec l'espoir que cela fasse l'effet d'une prise de conscience dans les populations en ayant montré que la liberté permise par le marché fini par l'aveuglement et le sentiment d'impuissance par rapport au fonctionnement global. Et cela est théoriquement justifiable. Je crains fort que cela relève d'un faux espoir que de croire que tous les acteurs adoptent le regard de l'observateur extérieur au système. Cela est d'autant plus improbable que si l'on suit Von Foerster dans sa conjecture, du point de vue des acteurs de l'intérieur le fonctionnement est perçu et ressenti objectivement comme leur échappant et animé comme par une main invisible. Cette objectivité de l'intérieur n'a ni plus ni moins de valeur que le point de vue de l'observateur extérieur. Il est donc inutile de vouloir opposer ces deux objectivités ou de vouloir prétendre à ce que tous les acteurs adoptent le point de vue de l'observateur extérieur d'autant plus que ce point de vue va s'opposer à la perception quotidienne animé par des ressentis.

\section{L'Etat comme régulateur?}

Une tentation pourrait alors d'amener le changement par l'édiction de nouvelles normes provenant de l'Etat ou du pouvoir politique. L'Etat entretient avec le citoyen une relation hiérarchique. Dans une démocratie, il entretient des boucles de rétroaction avec les citoyens. Dans ces boucles, de nombreuses informations se perdent. La capacité adaptative de l'Etat est relativement limitée. Il ne peut à lui seul répondre aux variations provenant de l'environnement. Comme le dit Godbout : "L'Etat est une hiérarchie, mais inclusive, non enchevêtrée, sans boucle, si ce n'est la boucle simple minimale du feedback. Les appareils sont des sens uniques, ce qui évite certains problèmes (la rencontre et l'accident, les rapports de domination par le don, etc ...), mais diminue d'autant la souplesse du système. Tout ce qui circule passe dans un centre avant de repartir dans l'autre sens, y laissant d'ailleurs une partie de son contenu à chaque étape, ce qui fait que ce qui circule arrive beaucoup plus réduit qu'au départ. La seule possibilité de retour est le feedback, c'est-à-dire le fait que le système ne retienne de l'extérieur que ce qu'il veut bien retenir. Alors que dans la boucle étrange, l'extérieur impose des choses au système. Il y a interaction dynamique. Pour sa part, le marché est un réseau enchevêtré, mais non hiérarchique. C'est pourquoi c'est également une boucle simple. ${ }^{\prime 5}$

\footnotetext{
${ }^{5}$ Godbout, Jacques, and Alain Caillé. L' esprit du don. Nachdr. Sciences humaines et sociales 86. Paris: La Découverte Poche, 2010. P.281
} 
D'autre part, L'histoire récente nous a montré que la remise en question de la liberté individuelle animée par le paradigme du marché- a de nombreux effets pervers dont les conséquences sont bien pires. (voir les échecs de la planification dans les régimes totalitaires).

\section{Démocratie et réseaux informels ?}

Il est dans toutes les sociétés des interactions entre les acteurs qui se concrétisent par des liens qui ne répondent pas aux postulats de l'échange marchand. Ces relations sont celle qui animent la socialité primaire. La socialité primaire est animée par un paradigme différent de celui de l'échange marchand caractérisé par l'équivalence dans l'échange, l'indépendance des acteurs. Il s'agit ici de rappeler les principes du paradigme du don au sens maussien. Le paradigme du don n'est pas animé par le postulat de l'équivalence. Le paradigme du don anime la socialité primaire et les communautés. Certains auteurs ont voulu mettre en parallèle don et marché. Ce faisant, ils montrent que le don crée une dépendance entre les acteurs qui va à l'encontre de sa liberté individuelle. Pourtant, le don est avant tout animé par la liberté des acteurs de participer ou non au cycle du don. Dans un système animé par le don, les acteurs se lient entre eux volontairement et en dehors de tout contrat négocié. La recherche de l'équivalence dans l'échange ne fait pas partie de l'échange. Le don implique l'imprévisibilité et l'inconditionnalité. Le don n'est pas prescriptible et échappe à un pouvoir central. Le don est typiquement une forme d'échange paradoxal car il implique la liberté de l'acteur tout en le liant à d'autres par des "dettes mutuellement acceptées". Il ne relève ni de l'individualisme propre au marché, ni de l'holisme propre à une société totalitaire.

Le don introduit dans une société une forme d'incertitude qui n'est pas de nature angoissante comme peut l'être l'incertitude qui se manifeste dans l'imprédictibilité du futur.

Cela dit, cela ne donne toujours pas de piste pour un renouvellement démocratique. Par contre, je postule que notre fonctionnement social et le rapport qu'entretienne la société civile et l'état en étendant les règles du marché à tous les secteurs de la société finissent par empêcher toute possibilité d'émergence de l'innovation sociale. La prise de conscience essentielle est celle d'accepter le désordre comme source d'innovation en s'abstenant de vouloir institutionnaliser rapidement toute émergence des mouvements. Il s'agit de favoriser et faciliter l'émergence d'espace public au sens d'Habermas. Il s'agit de favoriser le développement associatif informel car une fois institutionnalisé ils perdent de leur spontanéité.

Ce qui est proposé ici relève de l'adoption d'une pensée et d'une action complexe. D'un point de vue idéologique, le communautarisme va privilégier le lien primaire propre au don. Ce type de lien exclu logiquement l'étranger. Du point de vue du marché, les relations entre les acteurs sont gouvernées par l'échange basé sur l'équivalence dans lequel tous les acteurs ont accès peu importe leur origine communautaire. Une proposition sociale qui s'appuierait sur la théorie systémique et cybernétique inviterait au développement d'une société associant les deux formes de relations sans prétendre réduire l'une à l'autre. Cela implique une réforme de l'éducation qui rendrait le citoyen capable de prendre de la distance par rapport à la perception des phénomènes et dans l'évaluation des situations qui se présentent à lui mais également acteur et participant de l'espace public. Cela implique également une réforme de la gouvernance démocratique qui serait à l'écoute de l'association de fait, des mouvements sociaux et qui résisterait à la tentation de l'institutionnalisation.

Ce qui va contre cette possibilité : l'institutionnalisation des services à la population, la contractualisation et la professionnalisation des fonctions socialisatrices sont des facteurs inquiétant et qui vont à l'encontre de l'expression démocratique. Le soupçon vis-à-vis de toute forme d'organisation non institutionnelle est un autre facteur. La spécialisation des services d'aides rend aveugle. 
Ce que l'on observe et qui est source d'espoir : G1000, indignés, soutien aux immigrés, Nuits-Debouts sont autant de mouvements qui marquent l'actualité et qui sont animés de l'intérieur en grande partie par des relations basées sur l'aspiration au paradigme du don. Ces mouvements ont une identité fragile qui se transforme dès qu'ils s'institutionnalisent. Les réseaux locaux d'échanges de savoirs et de services, les associations de voisins, les différentes formes d'économies locales qui sont autant d'organisations qui sont fondées en grande partie sur un fonctionnement informel.

Ce que l'on observe et qui est contreproductif : des mouvement politiques animés par des personnes qui ressentent légitimement que le fonctionnement du système leur échappe et qui réifie ce fonctionnement en identifiant un groupe ou une communauté comme étant aux commandes alors qu'il s'agit d'une forme d'auto-organisation. Des décisions politiques maladroites qui visent à institutionnaliser les associations dont le fonctionnement est communautaire. Les associations ellesmêmes qui sont dans l'illusion qu'en institutionnalisant leur fonctionnement elles ne changent pas d'identité. Et enfin le citoyen qui ne s'éduque pas à une distanciation par rapport aux situations vécues.

\section{En conclusion}

La démocratie étant un régime qui se fonde sur la diversité et le conflit (sans violence) est le seul régime qui permet le développement social et individuel. Là où la démocratie actuelle montre ses limites est qu'elle adopte de manière uniforme le paradigme de l'échange marchand aussi bien dans les évaluations, les décisions, les discours et l'action.

Au-delà des modalités institutionnelles d'application du régime démocratique (vote, élection, tirage au sort, ...), il est intéressant de se questionner sur le rapport éventuel entre les relations entre les éléments et la totalité qui en émerge. Le challenge est de développer la capacité au sein de la population d'agir et de choisir au sein d'une dialogique.

Ce que j'ai tenté de montré dans cet article, c'est que la domination de l'échange marchand introduit des dysfonctionnements. Ce diagnostic de dysfonctionnement n'est pas justifié par une position idéologique mais par le fait que l'échange marchand lie les acteurs dans des relations rigides, prédictibles qui les rend aveugle et impuissant face au fonctionnement global. Si ce développement rejoint des positions idéologiques déjà présente dans le paysage politique le fondement n'est pas basé sur un jugement de valeur mais sur l'application d'un simple théorème issue de la cybernétique. Cela dépassionnalise un débat éventuel mais cela ne donne pas plus de valeur de vérité. La conjecture ouvre cependant des pistes d'actions possibles aussi bien au citoyen qui participant dans l'espace public gagne en influence sur le fonctionnement pour peu que le politique et l'associatif résiste à la tentation d'y projeter le modèle de l'échange marchand et/ou de l'institutionnalisation.

Comme Alain Caillé le commente : "Retenons-en qu'il existe un lien privilégié entre association et démocratie. Que, comme le don et le politique, ils sont une seule et même chose, mais à une échelle différente. Cette hypothèse est de nature à lancer l'interrogation sur la place de la démocratie dans nos sociétés sur des pistes nouvelles. Car le sort de la démocratie ne se joue pas seulement au niveau d'un pacte collectif tacite, au niveau politique ; pas seulement au niveau de la politique instituée, au niveau de ce qu'on pourrait appeler les espaces publics secondaires. Elle se jouent aussi, et peut-être d'abord, au sein de ces espaces publics primaires que constituent les associations."

"Les sociétés en voie de mondialisation ne renoueront avec un démocratie effective - au-delà du libre choix offert au consommateur et de la jouissance de protections sociales minimales - que si un nombre significatif d'associations variées permettent aux individus d'expérimenter des formes de vivre-

\footnotetext{
${ }^{6}$ Alain Caillé. Anthropologie du don: le tiers paradigme. Paris: Découverte, 2007. P.134
} 
ensemble autonomes tant vis-à-vis de la logique du marché que de celle de l'administration, et capable de contester leur hégémonie au nom de cette autonomie. Réciproquement, l'Etat comme le marché ont en fait besoin de cette force de contestation qui les empêche de devenir autiste et bêtes. Face aux incertitudes et à la faiblesse de la militance spontanée, il est permis de se demander si l'Etat et les partis politiques n'auraient pas bizarrement intérêt - un intérêt éclairé et bien compris à long terme - à tout faire pour susciter cette autonomie."7

Si cela semble une tâche inaccessible, je suis optimiste quant au fonctionnement actuel d'une partie de la jeune génération. Elle semble se défaire de deux mythes qui animent encore probablement l'ancienne génération : l'idéologie et le leader d'opinion. J'observe qu'une franche de la jeunesse n'est plus en quête d'un leader et qu'elle ne se situe plus dans la démarche idéologique. Cette tendance est favorable au fonctionnement en réseau et à la décentralisation. Ce qui peut paraître comme un désintérêt pour la politique et les affaires publiques peut également être interpréter comme l'émergence d'une nouvelle manière de s'organiser collectivement qui nous échappe encore.

\section{Références}

Andreewsky, Evelyne, and Robert Delorme, eds. Seconde Cybernétique et Complexité: Rencontres Avec Heinz von Foerster. Collection Ingénium. Paris: L'Harmattan, 2006.

Minati, Gianfranco. Towards a Post-Bertalanffy Systemics. New York, NY: Springer Science+Business Media, 2015.

Gianfranco Minati. Collective Beings. Contemporary Systems Thinking. Boston, MA: Springer US, 2006.

Chanial, Philippe, ed. La Société Vue Du Don: Manuel de Sociologie Anti-Utilitariste Appliquée. Textes à L'appui. La Bibliothèque Du MAUSS. Paris: La Découverte, 2008.

Godbout, Jacques, and Alain Caillé. L' esprit du don. Nachdr. Sciences humaines et sociales 86. Paris: La Découverte Poche, 2010.

Alain Caillé. Anthropologie du don: le tiers paradigme. Paris: Découverte, 2007.

\footnotetext{
${ }^{7}$ Chanial, Philippe, ed. La Société Vue Du Don: Manuel de Sociologie Anti-Utilitariste Appliquée. Textes à L'appui. La Bibliothèque Du MAUSS. Paris: La Découverte, 2008 p.362
} 
